\title{
Effects of glide slope, noise intensity, and noise duration on the extrapolation of FM glides through noise
}

\author{
KEITH R. KLUENDER and RICK L. JENISON \\ University of Wisconsin, Madison, Wisconsin
}

\begin{abstract}
Listeners are quite adept at maintaining integrated perceptual events in environments that are frequently noisy. Three experiments were conducted to assess the mechanisms by which listeners maintain continuity for upward sinusoidal glides that are interrupted by a period of broadband noise. The first two experiments used stimulus complexes consisting of three parts: prenoise glide, broadband noise interval, and postnoise glide. For a given prenoise glide and noise interval, the subject's task was to adjust the onset frequency of a same-slope postnoise glide so that, together with the prenoise glide and noise, the complex sounded as "smooth and continuous as possible." The slope of the glides $(1.67,3.33,5$, and $6.67 \mathrm{Bark} / \mathrm{sec})$ as well as the duration $(50,200$, and $350 \mathrm{msec})$ and relative level of the interrupting noise $(0,-6$, and $-12 \mathrm{~dB} \mathrm{~S} / \mathrm{N})$ were varied. For all but the shallowest glides, subjects consistently adjusted the offset portion of the glide to frequencies lower than predicted by accurate interpolation of the prenoise portion. Curiously, for the shallowest glides, subjects consistently selected postnoise glide onset-frequency values higher than predicted by accurate extrapolation of the prenoise glide. There was no effect of noise level on subjects' adjustments in the first two experiments. The third experiment used a signal detection task to measure the phenomenal experience of continuity through the noise. Frequency glides were either present or absent during the noise for stimuli like those used in the first two experiments as well as for stimuli that had no prenoise or postnoise glides. Subjects were more likely to report the presence of glides in the noise when none occurred (false positives) when noise was shorter or of greater relative level and when glides were present adjacent to the noise.
\end{abstract}

In most natural situations, the sound source to which one is attending may not be the only source present and interruptions by other sounds are frequent. The problem for perception lies in the fact that the influences of all sources are summed in the pressure wave that reaches the ear. Despite this complication, listeners usually have little difficulty keeping sources perceptually distinct and continuous despite interference and interruption. Investigations of phenomena such as auditory induction (e.g., Warren, 1984) and primary auditory stream segregation (e.g., Bregman, 1981, 1990) document the impressive ability of listeners to organize their auditory world in the face of such complications.

In the case of auditory induction, when portions of an acoustic signal are deleted and replaced with noise- of sufficient relative intensity and of appropriate spectral composition to have masked the signal were it presentlisteners perceive the signal as continuous (Warren, 1984). In this fashion, listeners seem to reconstruct the signal to maintain a coherent auditory world. Although the con-

This work was supported by NIDCD Grant DCO0719. The authors are grateful to Andrew Lotto and to three anonymous reviewers for very helpful comments on an earlier version of this paper. Address correspondence to Keith R. Kluender, Department of Psychology, University of Wisconsin, 1202 West Johnson Street, Madison, WI 53706. ditions under which perceptual continuity is maintained in tasks of auditory induction and streaming have been the subject of considerable investigation, the mechanisms responsible are not well understood.

Ciocca and Bregman (1987) have suggested that the two cues, "frequency proximity" and "alignment on a common trajectory"- which are analogous to the Gestalt principles of proximity and good continuity-together appear to be adequate to describe their findings in studies of auditory induction for gliding and steady-state tones. They conducted a series of experiments with stimuli consisting of a single interval of broadband noise preceded and followed by a sine component consisting of a glide with logarithmically increasing frequency, a glide with logarithmically decreasing frequency, or a sine wave of constant frequency. Although in much of the past work (Warren, 1984) the dependent variable used to assess the degree of induction was either the minimum amplitude or maximum duration of noise through which the listener could perceive a sound as continuous, Ciocca and Bregman (1987) used only a single noise duration (150 or $200 \mathrm{msec}$ ) and relative level $(-20 \mathrm{~dB} S / N)$ and asked listeners to rate the degree to which the tone sounded continuous after having heard four repetitions of one of these ensembles.

Ciocca and Bregman's (1987) third experiment is somewhat similar to the studies to be reported here. In that experiment, they used a 200-msec noise and both prenoise 
and postnoise sounds were descending glides of the same slope (either steep or shallow). Postnoise glides had starting frequencies lower than, higher than, and equal to the frequency preditted by accurate interpolation of the initial glide across the noise. Across conditions in their first two experiments, Ciocca and Bregman found that subjects were more likely to report stimuli as sounding continuous when the onset frequency of the signal (tone or glide) following the noise matched the offset frequency of the prenoise signal (proximity) and when the slope of the following glide was consistent with the trajectory of the prenoise glide (trajectory). Yet in their third experiment, they found that subjects heard these stimuli as sounding most continuous when the postnoise glide had a frequency onset consistent with the trajectory of the first glide, and they noted the absence of a proximity effect.

Although no clear effect of proximity was apparent in Ciocca and Bregman's (1987) third experiment, their null result seems to be at odds with an earlier study (Pollack, 1977) in which such an effect was observed, albeit in experiments using quite different materials and judgments. Pollack (1977) asked how well a listener could "extrapolate an auditory temporal gradient through a temporal gap." His stimuli consisted of a series of pulses that in the case of increasing frequency stimuli had successively shorter interpulse intervals and for decreasing frequency had successively longer interpulse intervals. Following the initial upward or downward frequency ramp, there was a period of silence $(0-8,000 \mathrm{msec})$ followed by another frequency ramp of the same slope. The task for the listener was to adjust the initial interpulse interval of the second ramp so that the pitch transition from the initial section sounded "as smooth as possible." With this experimental paradigm, Pollack found that underextrapolation was greater as the slopes of the ramps were greater and when the silent interval between ramps was longer.

The reason for the discrepant findings between these two studies (Ciocca \& Bregman, 1987; Pollack, 1977) is potentially quite important to theories regarding how listeners maintain an integrated auditory world. Ecologically relevant sounds are rarely of a monotonous nature. For speech sounds in particular, information is carried in the change in spectrum over time. Formant transitions signal both place and manner of articulation for consonants, and formant transitions define vowel diphthongs. A useful model of auditory integration must be especially informative with regard to signals such as speech, which change over time.

There are a number of potentially important differences between the Pollack (1977) and Ciocca and Bregman (1987) studies. First, there is the nature of the frequency ramps themselves (a train of pulses with changing interpulse intervals vs. a sine wave with continuously changing period), although there is no obvious reason why that difference should be particularly significant. Second, Pollack used silent intervals that were generally much longer than the noise intervals used by Ciocca and Bregman; however, close inspection of Pollack's published figures reveals clear underextrapolation for silent intervals $250 \mathrm{msec}$ and longer (although the data for a $100-\mathrm{msec}$ interval are equivocal). Perhaps the most important departure between these two studies is the nature of the task itself. In the Ciocca and Bregman study, the degree of illusory restoration through noise was the dependent variable. In the Pollack study, subjects adjusted the onset frequency of the pulse train following the silent gap, and there would be no perceptual restoration across the silence. Experiments described below are designed to further investigate the nature of perceptual continuity for stimuli changing in frequency.

\section{EXPERIMENT 1}

Experiment 1 shares important characteristics with the experiments of both Ciocca and Bregman (1987) and Pollack (1977). As in Pollack's studies, the method of adjustment was used. However, in this study, glides were separated by intervals of broadband noise varying from an RMS level equal to the level of the FM glides $(0 \mathrm{~dB} \mathrm{~S} / \mathrm{N})$ to a level $12 \mathrm{~dB}$ greater than the RMS level of the glides $(-12 \mathrm{~dB} S / \mathrm{N})$, whereas Pollack used a silent interval between the frequency glides and Ciocca and Bregman used one level of noise $(-20 \mathrm{~dB} S / \mathrm{N})$. On the basis of earlier studies (Warren, 1984), it could be assumed that the lower relative intensity noise $(0 \mathrm{~dB} / \mathrm{S})$ would be unlikely to give rise to illusory restoration, and this condition would be most analogous to the silent intervals used by Pollack. In contrast, the higher relative intensity noise interval $(-12 \mathrm{~dB} \mathrm{~S} / \mathrm{N})$ would most clearly result in perception of a continuous restored glide and would be most analogous to the studies by Ciocca and Bregman.

Upward frequency glides of four different slopes were used, and in a departure from Ciocca and Bregman (1987), who used logarithmic FM glides, and Pollack (1977), who used both linear and logarithmic glides, the frequency change was scaled in Bark-rate units. Bark-rate units, a psychophysically derived scale based upon critical bandwidths (Zwicker \& Terhardt, 1980), were chosen because they better approximate the psychological dimension of pitch.

\section{Method}

Subjects. Subjects were 12 undergraduate and graduate students at the University of Wisconsin who were naive to the purposes of the experiment. All reported normal hearing, and each was paid $\$ 10$ for participating.

Stimuli. Glides and intervening noise were digitally constructed with 16-bit resolution and 4-kHz sampling rate using software designed for this experiment on an $\mathbf{8 0 3 8 6}$ microcomputer. Upward glides were constructed according to the equation: $z_{c} /$ Bark $=$ $13 \arctan (0.76 f)$, where $z_{c}$ is critical bandrate in Bark, arctan is in radians, and $f$ is frequency in kilohertz (Zwicker \& Terhardt, 1980). A schematic representation of the stimuli is shown in Figure 1. The total stimulus complexes were $1,200 \mathrm{msec}$ in duration: 500 -msec prenoise glide, 200 -msec noise, and 500 -msec postnoise glide. For any given stimulus complex, both the prenoise and postnoise glides had the same slope $(1.67,3.33,5.00$, or 6.67 Bark/sec). The median frequency of the glides (which was deleted 


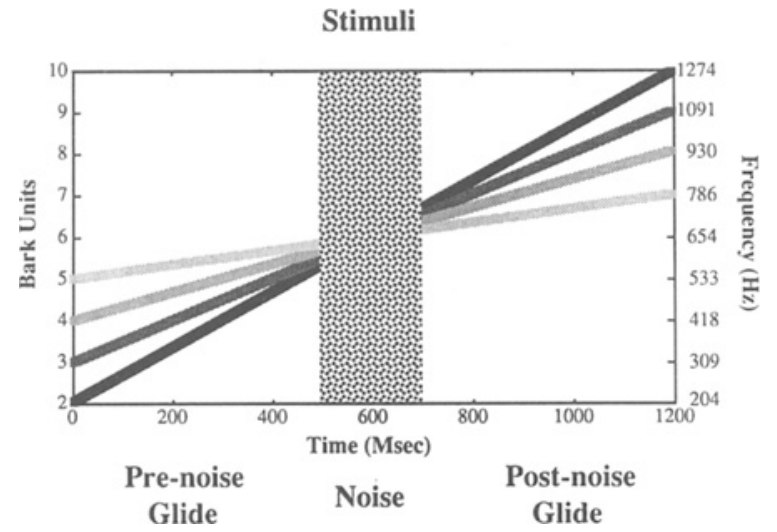

Figure 1. Schematic representations of stimuli used in Experiment 1. The four slopes used for all experiments reported $(1.67,3.33$, 5.00, and 6.67 Bark/sec) are indicated by the darkness of the lines. On any given trial, the same slope was used for the prenoise and postnoise glide.

and replaced by noise) was fixed at 6 Bark $(654 \mathrm{~Hz})$, so steeper glides began at a lower frequency and ended at a higher frequency than shallower glides. Bark and frequency values for the onset and offsets of the prenoise and postnoise glides (for accurate Bark extrapolation) are listed in Table 1. For each glide slope, nine different parallel postnoise glides were constructed at 0.50-Bark intervals, yielding a 4-Bark range in onset and offset frequencies ( \pm 2 Bark). The 200 -msec interrupting noise was digitally generated, rectangularsampled white noise with a $2-\mathrm{kHz}$ bandwidth and an RMS level 0,6 , or $12 \mathrm{~dB}$ greater than the RMS level of the glides ( $80 \mathrm{~dB}$ SPL). The onsets and offsets of the glide and noise portions of the stimulus complexes were cosine ramped over $5 \mathrm{msec}$. On a given trial, the prenoise glide and intervening noise remained constant for the series of adjustments. On the first trial, the postnoise glide was chosen randomly from the nine possible postnoise glides. In response to the subject's buttonpress, a higher or lower postnoise glide was appended to the prenoise glide and noise. Following D/A conversion (Ariel DSP-16), stimuli were lowpass filtered (cutoff frequency $1.8 \mathrm{kHz}$ ) through a bank of two 24 -dB/octave filters in series (Krohn-Hite 3343), prior to being amplified (Stewart HDA4) and played over headphones (Beyer DT-100) at a level of $80 \mathrm{~dB}$ SPL for the glide portion.

Procedure. Each subject was nun separately in a single-subject soundproof booth (Suttle Equipment Corp.). For a given onset glide slope and relative noise level, the task for the subject was to adjust the frequency of the postnoise glide so that, together with the prenoise glide, the stimulus complex sounded "as smooth and continuous as possible." The subjects adjusted the postnoise glide by pressing either the left or right button on a three-button mouse. When the subjects pressed the left button, the stimulus complex was replayed with a postnoise glide beginning one step lower in frequency than the glide on the last trial. When the subjects pressed the right button, the complex was replayed using the next higher glide. The subjects were urged to make several passes above and below the combination that sounded as smooth and continuous as possible before pressing the center button on the mouse to record their uitimate satisfaction with their last adjustment. (Inspection of the subjects' trial-by-trial behavior confirmed that the subjects followed this instruction, usually passing over their final adjustment six or more times.) Each of the 12 conditions (four slopes $x$ three noise intensities) was presented 12 times for a total of 144 trials. The experiment was broken up into two sessions, with six repetitions of each condition in a session. Each session took about 25 min, depending upon the speed with which each subject made his/her judgments, and the subjects were given a 10-15 min break between sessions.

\section{Results}

Two of the 12 subjects could not do the task as presented, and their data were not submitted to statistical analysis. One subject was extremely variable in his responses from trial to trial, and a second subject consistently adjusted the postnoise glide to the highest position possible. As can be seen in Figure 2, the remaining subjects consistently adjusted the offset portion of the glide to frequencies higher than the offset frequency of the prenoise glide ( $P$ in Figure $2 a$ ), and for all but the shallowest glides they consistently adjusted the postnoise glide to frequencies lower than those predicted by extrapolation of the prenoise portion ( $\mathrm{T}$ in Figure 2a). The effect of glide slope was significant $[F(3,9)=20.85, p<.0001]$, with steeper slopes resulting in greater underestimation of the glide trajectory. Curiously, the subjects adjusted the postnoise glide to a value that represents an overestimation of the glide trajectory when the slope was shallowest (1.67 Bark/sec). Perhaps more striking is the fact that the relative level of the noise had practically no effect on the subjects' adjustments $[F(2,9)=0.57]$ or upon the variance of the judgments.

\section{Discussion}

There are several notable aspects to the results of Experiment 1. First, the subjects evidence appreciable under-

Table 1

\begin{tabular}{|c|c|c|c|c|c|c|c|c|c|c|c|c|c|}
\hline \multirow{3}{*}{ (Bark } & \multirow{3}{*}{$\begin{array}{l}\text { Slope } \\
\text { per second) }\end{array}$} & \multirow{2}{*}{\multicolumn{2}{|c|}{ Onset }} & \multirow{2}{*}{\multicolumn{2}{|c|}{ Offset }} & \multirow{2}{*}{\multicolumn{2}{|c|}{ Onset }} & \multirow{2}{*}{\multicolumn{2}{|c|}{ Offset }} & \multicolumn{4}{|c|}{ Range } \\
\hline & & & & & & & & & & \multicolumn{2}{|c|}{ Onset } & \multicolumn{2}{|c|}{ Offset } \\
\hline & & Bark & Hertz & Bark & Hertz & Bark & Hertz & Bark & Hertz & Bark & Hertz & Bark & Hertz \\
\hline & 1.67 & 5.00 & 533 & 5.84 & 624 & 6.17 & 676 & 7.00 & 786 & $\begin{array}{l}4.17 \\
8.17\end{array}$ & $\begin{array}{l}437 \\
956\end{array}$ & $\begin{array}{l}5.00 \\
9.00\end{array}$ & $\begin{array}{r}533 \\
1091\end{array}$ \\
\hline & 3.33 & 4.00 & 418 & 5.67 & 613 & 6.33 & 696 & 8.00 & 930 & $\begin{array}{l}4.33 \\
8.33\end{array}$ & $\begin{array}{l}455 \\
981\end{array}$ & $\begin{array}{r}6.00 \\
10.00\end{array}$ & $\begin{array}{r}654 \\
1274\end{array}$ \\
\hline & 5.00 & 3.00 & 309 & 5.50 & 593 & 6.50 & 719 & 9.00 & 1091 & $\begin{array}{l}4.50 \\
8.50\end{array}$ & $\begin{array}{r}475 \\
1008\end{array}$ & $\begin{array}{r}7.00 \\
11.00\end{array}$ & $\begin{array}{r}786 \\
1486\end{array}$ \\
\hline & 6.67 & 2.00 & 204 & 5.34 & 573 & 6.67 & 741 & 10.00 & 1274 & $\begin{array}{l}4.67 \\
8.67 \\
\end{array}$ & $\begin{array}{r}494 \\
1036 \\
\end{array}$ & $\begin{array}{r}8.00 \\
12.00\end{array}$ & $\begin{array}{r}930 \\
1739\end{array}$ \\
\hline
\end{tabular}



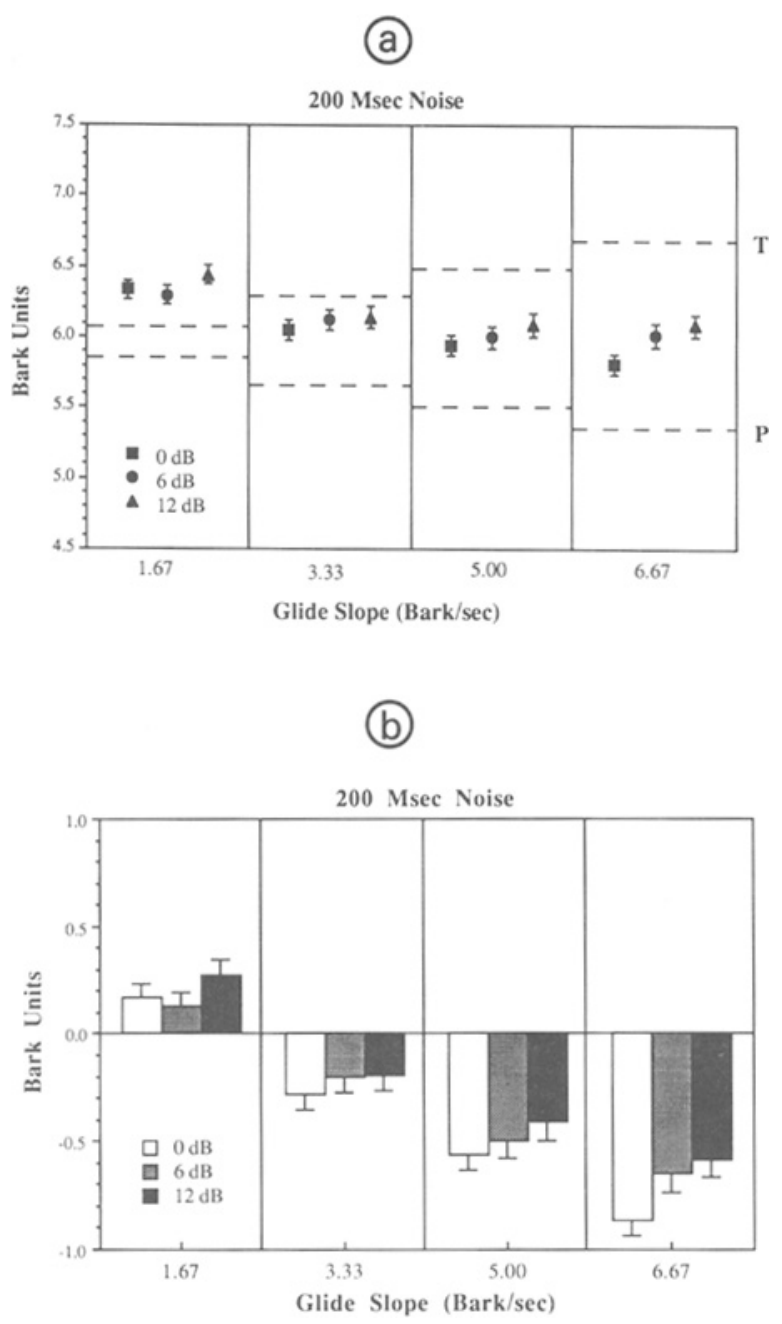

Figure 2. (a) Means and standard errors for adjustments in Bark units as a function of intervening noise intensity and glide slope. Ending frequencies of initial glides are represented by lower dotted lines labeled $P$ (proximity), and frequencies for accurate extrapolation of glides in Bark units are represented by upper dotted lines labeled $T$ (trajectory). (b) Means and standard errors for adjustments relative to accurate extrapolation in Bark units (designated 0.0 on the $x$-axis).

shoot in their adjustments for all but the shallowest glides. In a manner quite analogous to Pollack's (1977) findings, this undershoot increased monotonically with increasing glide slope. However, the subjects showed overshoot in their adjustments with the shallowest glides (1.67 Bark/ $\mathrm{sec})$. Finally, the relative level of the noise had no effect.

\section{EXPERIMENT 2}

In Pollack's earlier auditory study (1977), it was found that the magnitude of the underestimation of the appropriate frequency starting point for the postsilence section was greater not only when the frequency transitions had greater slopes but also when the intervening silent interval was longer. In Experiment 2, the effect of intervening noise duration was evaluated.

\section{Method \\ Subjects. Subjects were 8 undergraduate and graduate students} at the University of Wisconsin who participated in Experiment 1 and who remained naive to the purposes of the experiment. All reported normal hearing, and each was paid $\$ 10$ for participating.

Stimuli. Stimuli were strictly analogous to those used in Experiment 1 except that instead of having $200 \mathrm{msec}$ of noise intervening between the two glide portions, 50 and $350 \mathrm{msec}$ of noise were used. These noise intervals were again made up of digitally generated, rectangular-sampled white noise with an RMS level 0,6 , or $12 \mathrm{~dB}$ greater than the RMS level of the glides. As in Experiment 1, the total stimulus complexes (prenoise glide, noise, postnoise glide) were $1,200 \mathrm{msec}$ in duration so that when the noise was $50 \mathrm{msec}$ in duration the adjacent glides were each $575 \mathrm{msec}$ long and when the noise was $350 \mathrm{msec}$ in duration the adjacent glides were each $425 \mathrm{msec}$ long. As such, noise duration is confounded with glide duration and frequency extent; however, this was considered to be more appropriate than using equal-length glides whereby noise duration would be confounded with overall stimulus duration. All four of the glide slopes from Experiment 1 were used in Experiment 2 $(1.67,3.33,5.00$, or $6.67 \mathrm{Bark} / \mathrm{sec})$, and onset frequencies of the first glide component and offset frequencies of the second glide component were the same as well. For stimuli with 50 -msec noise portions, the offset frequencies of the prenoise glides were 649,644 , 639 , and $634 \mathrm{~Hz}$, and for accurate extrapolation in Bark-rate units, onset frequencies of the postnoise glides were $660,665,670$, and $676 \mathrm{~Hz}$. For stimuli with 350 -msec noise portions, the offset frequencies of the prenoise glides were $618,582,547$, and $513 \mathrm{~Hz}$, and the extrapolated onset frequencies of the postnoise glides were $692,729,769$, and $809 \mathrm{~Hz}$. As before, for each glide slope, nine different parallel postnoise glides were constructed at 0.50-Bark intervals. All other stimulus and presentation details were the same as in Experiment 1.

Procedure. The task and instructions for Experiment 2 were the same as those for Experiment 1. The subjects listened to stimuli with each noise interval in four separate sessions. Half of the subjects listened to stimuli with 50 -msec noise intervals during the first and third sessions, while the other half listened to stimuli with 350msec noise intervals during the first and third sessions.

\section{Results}

Figure 3 displays data for both the 50-msec and 350msec noise intervals. As was the case in Experiment 1, for all but the shallowest glides, the subjects consistently adjusted the offset portion of the glide to frequencies lower than those predicted by extrapolation of the prenoise portion. Again, underestimation was greater with steeper glide slopes $[F(3,7)=13.83, p<.0001]$, and when the slope was shallowest $(1.67 \mathrm{Bark} / \mathrm{sec})$, the subjects again adjusted the postnoise glide to a value that represents an overestimation of the glide trajectory. Since this overestimation was greater when the noise was short $(50 \mathrm{msec})$, the difference between the overall means (across slope) for 50- and 350-msec noise conditions did not reach statistical significance $[F(1,7)=2.49, p=.16]$. Consequently, the effect of noise duration is statistically manifested as a significant interaction between noise duration and slope $[F(3,7)=3.70, p<.05]$. Comparing the data from Experiments 1 and 2, it appears that the degree of undershoot is generally smaller in Experiment 2, and this is 
(a)

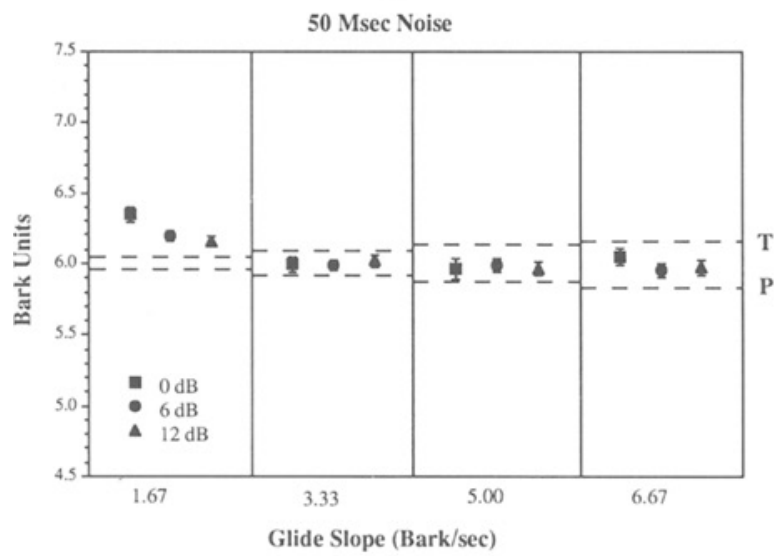

(C)

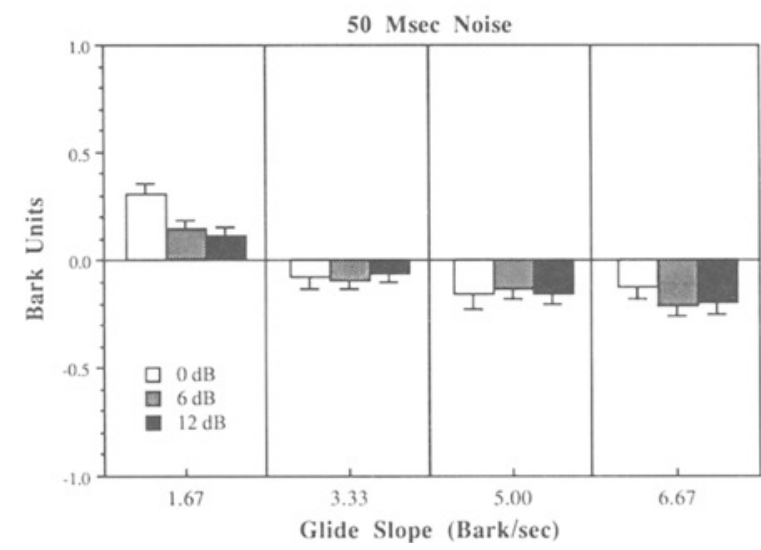

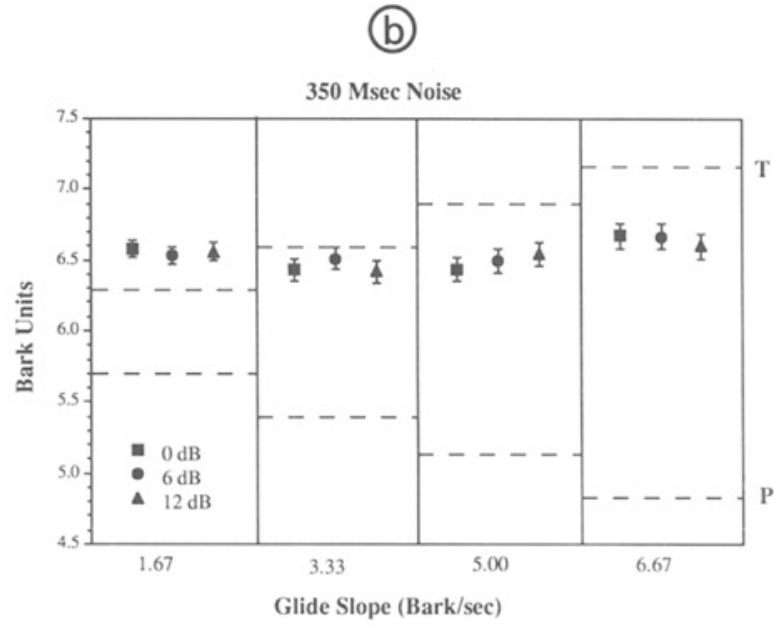

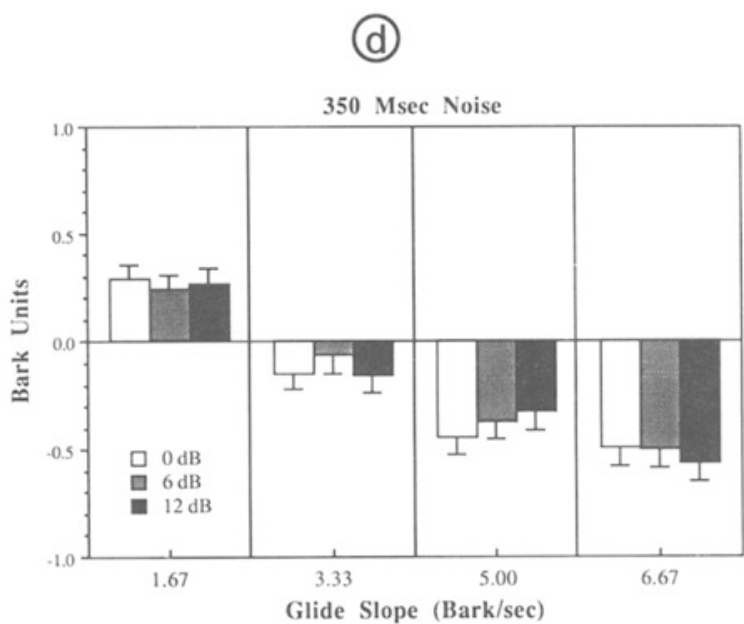

Figure 3. (a and b) Means and standard errors for adjustments in Bark units as a function of intervening noise intensity and glide slope. Adjustments for 50- and 350-msec noise durations are displayed in a and b, respectively. Ending frequencies of initial glides are represented by lower dotted lines labeled $\mathbf{P}$ (proximity), and frequencies for accurate extrapolation of glides in Bark units are represented by upper dotted lines labeled $T$ (trajectory). ( $c$ and d) Means and standard errors for adjustments relative to accurate extrapolation in Bark units (designated 0.0 on the $x$-axis). Adjustments for 50 and 350 -msec noise durations are displayed in $c$ and d, respectively.

likely due to the practice the subjects received in Experiment 1 . Of course, no interpretable statistical comparison can be made between Experiments 1 and 2 that takes into account practice and order effects. Again, the relative level of the noise had no effect on the subjects' adjustments $[F(2,7)=0.05, p=.96]$.

\section{Discussion}

Experiment 2 served to replicate the essential findings from Experiment 1, while extending these observations to shorter and longer noise intervals. In addition, an effect of intervening noise duration was found that is consistent with Pollack's (1977) auditory experiments. The finding that subjects actually seem to overestimate the trajectory with the lowest slopes was replicated as well. Again, there was a clear lack of effect when the relative level of the noise varied from high levels at which the glides should sound continuous through the noise to lower levels at which the glides sound interrupted. Experiment 3 further addresses this finding.

\section{EXPERIMENT 3}

Given that one of the major differences between the earlier studies (Ciocca \& Bregman, 1987; Pollack, 1977) was whether silence or noise intervened between the glide portions, the conspicuous lack of an effect of relative noise level-presumably manifested as perceived contiguity-is somewhat interesting. It is not clear whether subjects should be better or worse at the task when the phenomenal experience is that of a continuous glide through the noise, but one might well imagine some effect for such a change in perception. This issue is directly relevant to the studies by Ciocca and Bregman (1987) in which perceived continuity was used as the dependent measure of best trajectory. For stimuli used in Experiments 1 and 2 above, both 
experimenters (KRK and RLJ) heard the $0 \mathrm{~dB}$ S/N complexes as sounding clearly discontinuous and heard the $-12 \mathrm{~dB}$ S/N complexes as clearly continuous. Furthermore, results frơm earlier" studies (e.g., Warren, 1984; Warren, Obusek, \& Ackroff, 1972) support the conclusion that for the $0 \mathrm{~dB} \mathrm{~S} / \mathrm{N}$ complexes, glides would be perceived as stopping and starting adjacent to the noise, and for the $-12 \mathrm{~dB} S / \mathrm{N}$ complexes, glides would be perceived as continuous through the noise.

In Experiment 3, the phenomenal experience of continuity was directly assessed using a signal detection task in which subjects were asked simply to report whether the frequency glide was present or not present in the noise. The subjects made these judgments for stimuli like those used in Experiments 1 and 2 as well as for stimuli with no prenoise or postnoise glides. The second condition (no prenoise and postnoise glides) was run to evaluate separately the contribution of adjacent glides to the perception of the frequency glide within the noise interval.

\section{Method}

Subjects. Subjects were 9 undergraduate and graduate students at the University of Wisconsin who had not participated in Experiments 1 and 2 and who were naive to the purposes of the experiment. All reported normal hearing, and each was paid \$20 for participating.

Stimuli. The same four glide slopes, three noise durations, and three noise intensities used in Experiments 1 and 2 were used in Experiment 3. For stimuli with prenoise and postnoise glides, the postnoise glide that constituted the accurate extrapolation (on the Bark-rate scale) of the prenoise glide was used. Half of the stimuli had no signal embedded in the noise as was the case in Experiments 1 and 2 , and half had a 1.67, 3.33, 5.00, or 6.67 Bark/sec glide embedded in the noise. The embedded glide had beginning and end- ing frequencies reflecting an accurate interpolation between the prenoise and postnoise glides. The same embedded glides were used for stimuli with and without prenoise and postnoise glides. This yielded 144 different stimuli (four glide slopes, three noise durations, three relative noise levels, signal present/absent, glides adjacent to noise present/absent; or $4 \times 3 \times 3 \times 2 \times 2$ ). As in Experiments 1 and 2 , stimuli were digitally constructed with 16-bit resolution and 4-kHz sampling rate using software designed for this experiment on a microcomputer.

Procedure. Each subject was run separately in a soundproof booth (Suttle Equipment Corp.) and listened to both types of stimuliwith and without glides adjacent to the noise-in four separate sessions of 360 trials (36 stimuli each presented 10 times in random order). Prior to each session, the subjects were presented with a visual characterization of the stimuli to be used in that session and were told that their task was to report the presence or absence of the tone glide within the noise by pressing buttons labeled "present" and "not present" on a response box. Brief $1 / 2-\sec$ warning lights above the response buttons flashed to signal the beginning of a trial, and the stimulus was presented immediately after the waming lights were turned off. The warning lights were turned back on following stimulus presentation and were turned off when the subject pressed a response button. There were approximately $2 \mathrm{sec}$ between trials, and each session lasted about $25 \mathrm{~min}$.

\section{Results}

For each subject, $d^{\prime}$ values were calculated for each glide slope, noise duration, and noise level. In conditions for which the subjects had all hits or all false positives, probabilities of .9999 or .0001 ( $\pm 3.2 \sigma)$, respectively, were designated. ${ }^{1}$ Performance of the nine subjects is presented in Figure 4. Analysis of variance revealed a number of significant main effects. As would be expected, the subjects were better at discriminating the presence or absence of embedded glides when the noise was longer

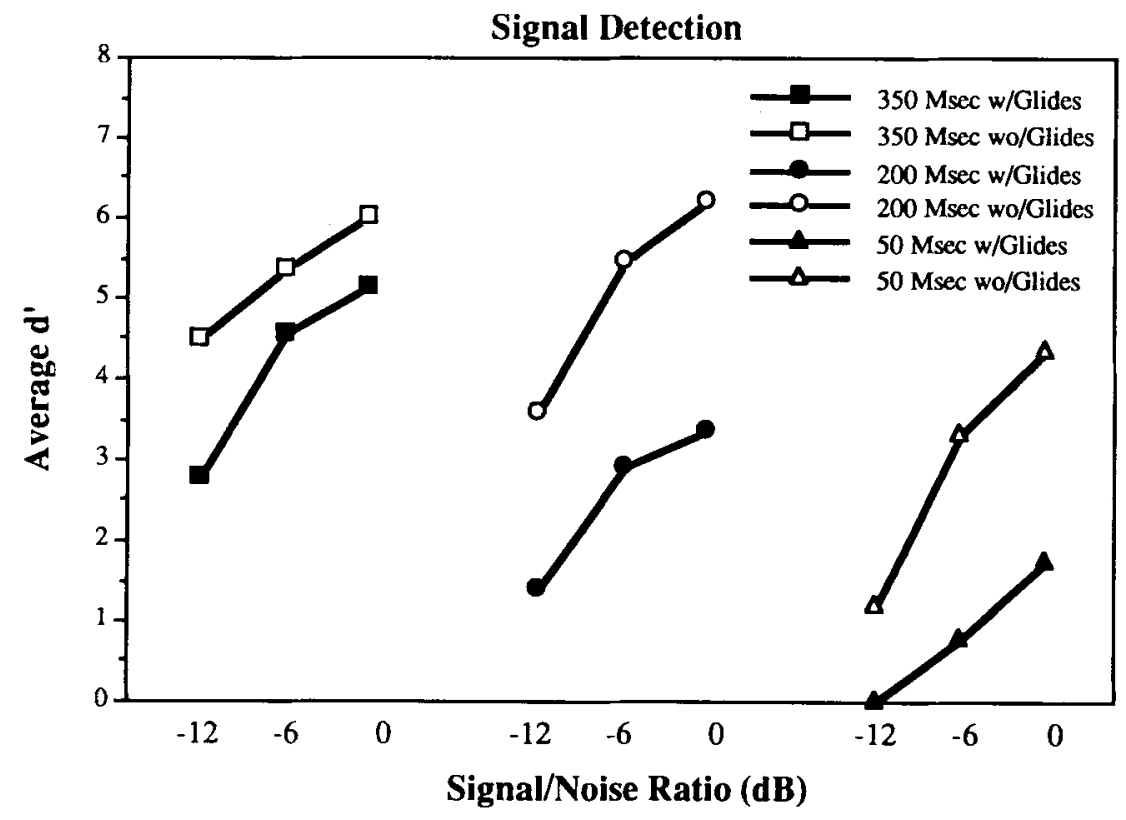

Figure 4. Average $d^{\prime}$ values plotted as a function of noise duration and intensity. Filled symbols represent judgments when adjacent glides are present. 
$[F(2,8)=84.08, p<.0001]$ or less intense $[F(2,8)=$ $184.98, p<.0001]$. The subjects were also more accurate when there were no glides adjacent to the noise $[F(1,8)=25.67, p<.01]$. There was no main effect for the slope of the glides $[F(3,8)=2.05]$. A significant interaction was found between the presence of adjacent glides and the relative level of the noise $[F(2,8)=6.60$, $p<.01$, probably as the result of a ceiling effect by which subjects perform extremely well at both of the lower noise intensities when there are no adjacent glides. A nonmonotonic interaction between noise level and slope was also statistically significant $[F(6,8)=3.16, p<.05]$. Finally, there was a significant three-way interaction between noise duration, relative noise level, and the presence of adjacent glides $[F(4,8)=4.24, p<.01]$.

\section{Discussion}

Especially when adjacent glides are present, subjects are more likely to perceive the signal as being present when it is not present if the relative level of the noise is $12 \mathrm{~dB}$ or even $6 \mathrm{~dB}$ higher than the glides and if the noise is relatively short. It is clear that subjects are more likely to perceive the glide as continuous through the noise when relative noise levels are greater $(-6$ or $-12 \mathrm{~dB} \mathrm{~S} / \mathrm{N})$ and when adjacent glides are present, yet there was no accompanying effect of relative noise level upon subjects' judgment of the appropriate postnoise glide starting point in Experiments 1 and 2. The phenomenal experience of continuity appears to neither enhance nor detract from subjects' ability to accurately judge the extrapolated trajectory of FM glides.

\section{GENERAL DISCUSSION}

What is clear across the first two experiments reported here is that, except for the case with the shallowest slopes, subjects use both frequency proximity and trajectory in judging the perceptual path of a signal changing in frequency. In fact, with the exception of the case for the shallowest glides, subjects seem to split the difference between proximity and trajectory by choosing postnoise onsets midway between the offset of the prenoise glide and the projected onset of the postnoise glide, although when the noise is $350 \mathrm{msec}$ in duration, trajectory seems to be the stronger influence.

Why did the subjects choose postnoise glides with higher onsets for the lowest slopes in Experiments 1 and 2 ? One hypothesis might be that the mix of four slopes within an experimental session leads to some sort of anchoring effect by which the underextrapolation in the three higher slope conditions gives rise to a bias in the opposite direction when slopes are low. However, since the subjects were not being asked to make dichotomous judgments such as high or low across trials, there should be no cause to overextrapolate on occasion to balance the predominance of underextrapolations. After all, the subjects were encouraged to be accurate on every trial, and they had no knowledge of whether they in fact made a high, low, or veridical choice on any given trial.

A second consideration may be that the Bark-rate scale, although arguably better than a log or linear scale, is not a perfect measure of pitch change for this task. However, it is not clear what, if any, scale would be more psychologically appropriate. If the Bark-rate scale was inappropriate by virtue of increasing more slowly than does the perception of pitch with the same change in frequency (i.e., something more akin to a log scale would have been more appropriate), then the "overshoot" for the low slope conditions may in fact be no overshoot at all. If the perception of pitch increases more rapidly than does the Barkrate scale, the overshoot could in fact be most accurate in some yet undefined psychological unit. It would then follow that the degree of undershoot in the conditions with greater slopes is underestimated using our stimuli since the "correct" extrapolation in Bark-rate units would always be too low relative to some more appropriate psychological units. Close inspection of Figures 2 and 3, however, shows that the subjects actually chose physically higher frequency starting points in the lowest slope condition, and no adjustment of the scale can change the fact that those adjustments are relatively higher than those for higher slopes.

It bears noting that the results from the present experiments are at least superficially analogous to the Poggendorff illusion for vision. For the visual case (an oblique transversal interrupted by parallel lines), subjects consistently underestimate the absolute trajectory of the transversal when making judgments of collinearity for the two transversal sections (Weintraub \& Krantz, 1971). Furthermore, this undershoot increases with increasing slope and with increasing distance between the transversals (Weintraub \& Krantz, 1971). Each of these effects can be seen in the first two experiments described above. Although others have stressed such analogies between audition and vision (e.g. , Julesz \& Hirsh, 1972), the hypothetical mapping between the two domains has been disputed (Handel, 1988a, 1988b; Kubovy, 1988), and the present authors simply report the analogy without theoretical comment.

Some researchers have doubted that trajectory is very important in maintaining auditory coherence (Bregman, 1990). For example, Deutsch's (1975) well-known scale illusion depends upon listeners failing to group successive tones on the basis of frequency trajectory (or even of presentation to the same ear) when an alternative organization can be formed on the basis of frequency proximity. There have been a number of additional studies reported suggesting that subjects may not be inclined to use trajectory information. For example, in auditorystream-segregation experiments in which multiple logfrequency glides can organize either by frequency proximity or frequency trajectory, little or no effect of trajectory was found (Steiger \& Bregman, 1981). Furthermore, Tougas and Bregman (1985) found that frequency trajectory as well as rhythmic regularity and glide duration had 
little effect upon stream segregation relative to frequency proximity. Finally, when Dannenbring (1976) carried out a study of auditory induction using linear-frequency glides that increased then decreased repetitively and he replaced the vertices with noise, subjects reported hearing the signal traverse the noise without extrapolating to the projected vertex. Of course, the null results of all of these studies are contrary to the significant effects of trajectory in the present experiments as well as in those by Ciocca and Bregman (1987).

Each of the studies that found little or no effect of trajectory, with the exception of the demonstration by Deutsch (1975), used repeating cycles as stimuli (Dannenbring, 1976; Steiger \& Bregman, 1981; Tougas \& Bregman, 1985), whereas this study as well as Ciocca and Bregman (1987) used single presentations of each stimulus complex. Given that auditory-stream segregation becomes stronger as the cycle is repeated (Bregman, 1978; van Noorden, 1975), this difference may be an important one. Of course, many ecologically significant sounds-speech, for example-do not repeat over and over, and one may well be reluctant to rule out the effect of trajectory only on the basis of null results from experiments with repetitive patterns.

\section{REFERENCES}

Bregman, A. S. (1978). Auditory streaming is cumulative. Journal of Experimental Psychology: Human Perception \& Performance, 4, 380-387.

Bregman, A. S. (1981). Asking the "What for" question in auditory perception. In M. Kubovy \& J. R. Pomerantz (Eds.), Perceptual organization (pp. 99-118). Hillsdale, NJ: Erlbaum.

Bregman, A. S. (1990). Auditory scene analysis. Cambridge: MIT Press.

Ciocca, V., \&regman, A. S. (1987). Perceived continuity of gliding and steady-state tones through interrupting noise. Perception \& Psychophysics, 12, 476-484.

Dannenbring, G. L. (1976). Perceived auditory continuity with alternatively rising and falling frequency transitions. Canadian Journal of Psychology, 30, 99-114.

Deutsch, D. (1975). Musical illusions. Scientific American, 233, 92-104.
HANDEL, S. (1988a). No one analogy is sufficient: Rejoinder to Kubovy. Joumal of Experimental Psychology: Human Perception \& Performance, 14, 321 .

HANDEL, S. (1988b). Space is to time as vision is to audition: Seductive but misleading. Joumal of Experimental Psychology: Human Perception \& Performance, 14, 315-317.

Julesz, B., \& HIRSH, I. J. (1972). Visual and auditory perception-An essay of comparison. In E. E. David \& P. Denes (Eds.), Human communication: A unified view (pp. 283-340). New York: McGraw-Hill.

KuBovy, M. (1988). Should we resist the seductiveness of the space: time:vision:audition analogy? Journal of Experimental Psychology: Human Perception \& Performance, 14, 318-320.

Pollack, I. (1977). Continuation of auditory frequency gradients across temporal breaks: The auditory Poggendorff. Perception \& Psychophysics, 21, 563-568.

Steiger, H., \& Brgman, A. S. (1981). Capturing frequency components of glided tones: Frequency separation, orientation, and alignment. Perception \& Psychophysics, 30, 425-435.

Tougas, Y., Bregman, A. S. (1985). The crossing of auditory streams. Jourmal of Experimental Psychology: Human Perception \& Performance, 11, 788-798.

VAN NOORDEN, L. P. A. S. (1975). Temporal coherence in the perception of tone sequences. Unpublished doctoral dissertation, Eindhoven University of Technology, Eindhoven, The Netherlands.

WARREN, R. M. (1984). Perceptual restoration of obliterated sounds. Psychological Bulletin, 96, 371-383.

WARREN, R. M., ObUSeK, C. J., \& ACkrofF, J. M. (1972). Auditory induction: Perceptual synthesis of absent sounds. Science, 176, 1149-1151.

Weintraub, D. J., \& Krantz, D. H. (1971). The Poggendorff illusion: Amputations, rotations, and other perturbations. Perception \& Psychophysics, 10, 257-264.

ZwICKER, E., TERHARDT, E. (1980). Analytical expressions for critical-band rate and critical bandwidth as a function of frequency. Joumal of the Acoustical Sociery of America, 68, 1523-1524.

\section{NOTE}

1. When there were 10 of 10 "hits" with no "false positives," $d$ ' was $6.400(2 \times 3.2 \sigma)$. Such cases are not unique to signal detection, since $100 \%$ or $0 \%$ accuracy is somewhat ambiguous to interpret using any measure of performance. A similar sort of truncation must be made, for example, when applying probit analysis, whereby one must truncate $0 \%$ and $100 \%$ scores on the tails.

(Manuscript received June 14, 1991; revision accepted for publication October 7, 1991.) 